\title{
ROBÓTICA COLABORATIVA EN APLICACIONES DE ASISTENCIA EN MÁQUINAS DE ELECTROEROSIÓN
}

\author{
Román Abad ${ }^{2}$, Asier Muñoz ${ }^{1}$, Olatz Flaño², Jose Mari Ramos², Aitziber Mancisidor ${ }^{1}$, Itziar Cabanes ${ }^{1}$ \\ roman.abad@ehu.eus,asier.munoz@ehu.eus, aitziber.mancisidor@ehu.eus, itziar.cabanes@ehu.eus \\ ${ }^{1}$ Departamento de Ingeniería de Sistemas y Automática \\ Escuela de Ingeniería de Bilbao, UPV/EHU \\ oflano@onaedm.com, jramos@onaedm.com \\ ${ }^{2}$ Ona Electroerosión S.A.
}

\begin{abstract}
Resumen
Los robots colaborativos, también denominados cobots, son robots industriales diseñados para dar respuesta a procesos de producción de alta variabilidad y mayor flexibilidad. Estos robots son muy adecuados para tareas de Machine Tending, permitiendo la automatización de los procesos de producción mediante tareas de asistencia a distintos tipos de máquina herramienta. En este trabajo se presenta el diseño de una célula robotizada donde se integra un robot colaborativo para dar respuesta a la necesidad de alimentación de electrodos en diferentes máquinas de electroerosión, sin parar el proceso de mecanizado y colaborando con las tareas a realizar por el usuario. Para la ejecución en simulación se ha implementado dicho escenario en la herramienta RoboDK.
\end{abstract}

Palabras clave: Robótica colaborativa, cobot, automatización, electroerosión, EDM, RoboDK.

\section{INTRODUCCIÓN}

La electroerosión es un proceso termoeléctrico en el que se elimina material de una pieza mediante una serie de descargas eléctricas generadas a alta frecuencia entre un electrodo y la pieza, encontrándose ambos sumergidos en un medio dieléctrico.

La principal ventaja de la electroerosión frente a otros procesos de fabricación es que la dureza del material no es una limitación. A su vez, al no haber contacto entre electrodo y pieza, se evitan problemas de chatter y vibraciones. La electroerosión es un proceso de fabricación en constante desarrollo, que permite producir piezas con geometrías muy variadas y complejas, destinadas a distintos sectores como el sector del molde o aeronáutica, entre otros [1].

Dentro de la electroerosión, se diferencian dos principales procesos: i) electroerosión por hilo o Wire
Electrical Discharge Machining (WEDM), proceso que permite el corte de geometrías complejas mediante un hilo conductor. ii) electroerosión por penetración o Sinking Electrical Discharge Machining (SEDM), proceso con el que mediante un electrodo (generalmente de grafito o cobre) se da a la pieza la forma deseada. Permite replicar la geometría del electrodo o generar una forma 3D mediante la programación de movimientos sincronizados.

En los últimos años, con la intención de dotar a las máquinas de WEDM y SEDM de mayor autonomía, a la vez de aumentar la productividad y el rendimiento, se ha empezado a utilizar robots para asistir en diferentes tareas de la electroerosión [8]. Este concepto de tareas de asistencia en máquina herramienta se unen bajo el concepto de "machine tending", que se define como la supervisión del trabajo de una máquina mientras realiza una tarea, incluyendo la alimentación de piezas [5]. Estas tareas, se pueden agrupar en dos bloques o categorías: por un lado, asistencia al proceso de fabricación; por otro lado, supervisión y mantenimiento [4].

Las tareas de asistencia al proceso de fabricación son tareas que participan activamente en el proceso, como por ejemplo la alimentación de piezas a la máquina. Normalmente, en el proceso de electroerosión cada pieza requiere de un tiempo de fabricación considerablemente alto. Esto implica que limitar las tareas de un robot únicamente a la alimentación de piezas supondría un tiempo de desaprovechamiento del mismo elevado. En cambio, la instalación de un robot colaborativo o cobot permitiría compartir el espacio de trabajo con otras personas y acercarse a ellas o a otras máquinas sin peligro de colisiones [2], [3], [7]. Además, la flexibilidad y facilidad de programación de un cobot permitiría automatizar diversas tareas con mayor aprovechamiento del mismo.

Unos de los primeros avances en máquinas SEDM fue la aparición de un intercambiador de electrodos incorporado en la máquina, capaz de retirar un electrodo de la máquina y sustituirlo por uno diferente 
desde un pequeño almacén [6]. Este avance ya podía dotar a las máquinas de una autonomía de hasta 24 horas. Sin embargo, esta autonomía podría verse incrementada si se automatiza también la alimentación de electrodos al intercambiador mediante un robot colaborativo.

Las tareas de supervisión y mantenimiento, son las encargadas de comprobar que el proceso de fabricación se realiza correctamente y, en caso de ocurrir una parada, detectarla para solucionar el problema. Las tareas de mantenimiento también incluyen modelos predictivos para anticiparse a posibles errores. Actualmente no se encuentran aplicaciones de estas características en la industria de la electroerosión, por lo que este es sin duda uno de los focos de interés en los que se centrarán los trabajos de investigación en el futuro.

Tras analizar las posibles tareas que podría realizar un cobot como asistente a máquinas de electroerosión, en el presente trabajo se plantea la simulación de tareas de alimentación de electrodos a diferentes máquinas de SEDM del fabricante ONA Electroerosión S. L.

El resto del artículo se estructura de la siguiente manera. En la sección 2 se presenta el diseño de la célula robotizada de machine tending en SEDM. En la sección 3, se detalla la implementación en simulación de la aplicación robotizada en un escenario compuesto por cuatro máquinas de electroerosión. En la sección 4, se realizará una discusión y análisis de los resultados, concluyendo en el apartado 5 con los aspectos más relevantes y trabajos futuros de esta aplicación.

\section{DISEÑO DE LA CÉLULA ROBOTIZADA}

La aplicación de la alimentación de electrodos en máquinas $E D M$ incluye diversas tareas que el cobot debe realizar. Es habitual que las máquinas dispongan de un pequeño almacén o intercambiador de electrodos situado junto a la misma (ver Figura 1). Desde este intercambiador la máquina es capaz de elegir el electrodo que sustentará su cabezal, y que será clave en la fabricación de la pieza. En función del tipo de máquina y de la complejidad de la pieza a mecanizar, los electrodos pueden ser iguales o tener formas y tamaños diferentes. Estos electrodos se van desgastando durante el proceso de electroerosión, por lo que necesitan ser reemplazados cada cierto tiempo. Una vez que los electrodos disponibles en el intercambiador han sido utilizados, deben ser reemplazados por electrodos nuevos para que la máquina pueda seguir funcionando, sin que se vea obligada a parar en ningún momento el proceso.
Con este propósito, se definen las siguientes tareas a realizar por el cobot:

- Recoger los electrodos desgastados del intercambiador de electrodos de la máquina.

- Llevar los electrodos utilizados a un depósito para su reparación o reciclaje.

- Coger electrodos nuevos, ya sea desde una mesa, un armario, una cinta transfer, etc.

- Colocar los electrodos nuevos en el intercambiador.

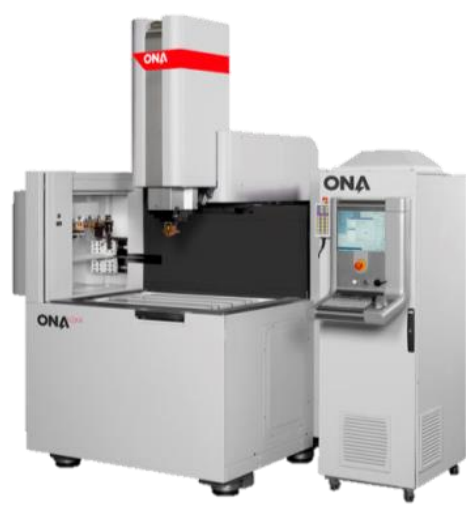

Figura 1: Máquina $Q X 4$ de ONA Electroerosión.

En cuanto a los requerimientos de carga y alcance del cobot, por un lado, se especifican electrodos de peso de hasta $10 \mathrm{~kg}$, y la ubicación del carrusel exige un alcance horizontal de un metro aproximadamente. El cobot modelo UR10e de Universal Robots, cumple con dichas especificaciones [9].

Para la realización de las diferentes tareas por parte del cobot, es necesario adaptar el escenario y dotarlo de elementos con los que el cobot pueda interactuar. Por un lado, es necesario añadir elementos a la máquina SEDM para que el cobot pueda controlar y asistir al carrusel de electrodos. Los elementos añadidos son una compuerta deslizante para permitir el acceso al carrusel, el giro de éste último y los botones de control que permiten su giro (ver Figura 2).

Además, se deben diseñar una serie de armarios desde los cuales el cobot sea capaz de coger electrodos nuevos y depositar los electrodos desgastados no útiles para el proceso. Para ello se utilizan métodos de agarre de tipo pinza en el armario.

Con la idea de que el cobot sea capaz de asistir a un mayor número de máquinas, se va a integrar sobre un robot móvil $(A M R)$ que permite desplazar el cobot libremente por la planta [10]. 


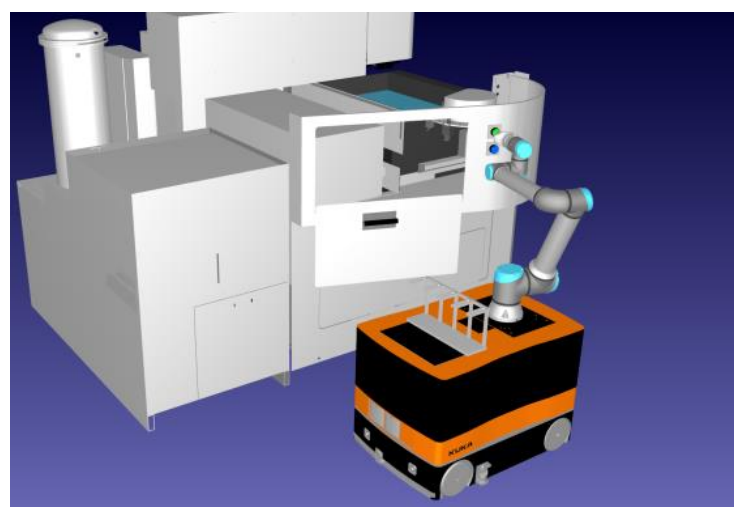

Figura 2: Robot colaborativo controlando el carrusel de una máquina de electroerosión $Q X 4$

\section{IMPLEMENTACIÓN}

\subsection{Caso de estudio 1}

Tras definir los elementos necesarios, se lleva a cabo la implementación de una planta de producción que contiene una máquina $Q X 4$ de ONA Electroerosión, un cobot URlOe de Universal Robots sobre un AMR, y dos armarios almacenes de electrodos, uno para recogida de electrodos nuevos y otro para depósito de electrodos desgastados (ver Figura 3).

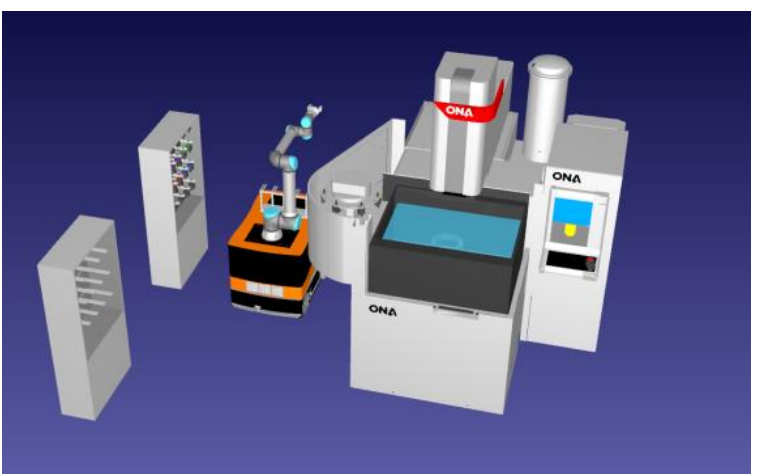

Figura 3: Escenario con una máquina SEDM

Una vez definido el escenario, se lleva a cabo la programación de la simulación en la herramienta RoboDK. Para ello se intercalan varias secuencias de carga y descarga de electrodos durante el proceso de mecanizado en la máquina. Cuando ésta detecta que es necesario un reemplazo en los electrodos del carrusel, el sistema envía una señal al cobot con los electrodos que es necesario sustituir y la posición en la que debe colocar cada uno. Como el ciclo de trabajo de la máquina con un mismo electrodo puede durar varias horas, el cobot tiene tiempo suficiente para realizar sus tareas sin interrumpir el trabajo de la máquina, demostrando que la tarea de asistencia en la alimentación de electrodos es una aplicación viable y adecuada para un robot colaborativo.

\subsection{Caso de estudio 2}

Dado que los electrodos, dependiendo de las condiciones, material y forma pueden tener una duración entre 10-90 minutos aproximadamente, se observa una gran diferencia con los tiempos invertidos para realizar los cambios de electrodos mediante el cobot, que es de unos minutos. Esto se traduce en que el cobot no es utilizado durante largos periodos de tiempo ya que no es requerido por la máquina. Por ello, se decide ampliar el escenario y se propone una planta más exigente, con cuatro máquinas $E D M$ (ver Figura 4), en la que el cobot es capaz de asistir a todas ellas mediante un sistema de tipo semáforo basado en prioridades en el que varias máquinas solicitan acceso a la utilización de un recurso compartido (cobot). El semáforo gestiona las distintas solicitudes y concede permiso de uso del cobot a la máquina más prioritaria.

El semáforo implementado es una herramienta que tiene dos ventajas principales. Por un lado, el semáforo tiene una cola en la que las tareas que están esperando acceden al recurso en función de la prioridad que se les haya asignado, y no por orden de petición de acceso al recurso. Por otro lado, una vez una máquina haya accedido al recurso, no puede ser desalojada hasta que finalice sus tareas, aunque una de mayor prioridad haya hecho una petición durante el tiempo de utilización. De esta manera, se consigue atender a las tareas más prioritarias primero, pero no se abortan las mismas mientras se están ejecutando. Con este sistema se reducen los trayectos del $A M R$ en los que realmente no está realizando una tarea útil y se optimiza el uso del cobot.

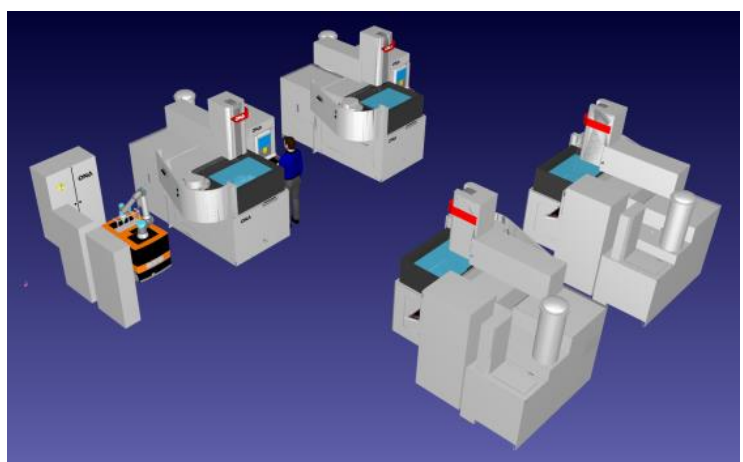

Figura 4: Escenario con diversas máquinas SEDM

\section{DISCUSIÓN Y RESULTADOS}

Para implementar el sistema basado en herencia de prioridades, cada máquina (i) dispone de tres tipos de señales booleanas: la primera tiene como objetivo mostrar cuándo una máquina está ejecutando código propio $\left(\mathrm{M}_{\mathrm{i}}\right)$, la segunda es una señal de petición de acceso al recurso compartido $\left(\mathrm{P}_{\mathrm{i}}\right)$, y la última muestra si la máquina está haciendo uso del recurso 
compartido $\left(\mathrm{U}_{\mathrm{i}}\right)$. Además de estas señales que tiene cada una de las máquinas, el cobot tiene una señal que refleja si está ejecutando alguna tarea $(C=T R U E)$ o si está disponible para realizar una nueva $(C=F A L S E)$. Las señales utilizadas se muestran en la Tabla 1.

Tabla 1: Señales utilizadas

\begin{tabular}{ll}
\hline DESCRIPCIÓN & NOMBRE SEÑNL \\
\hline Código propio & $\mathrm{M}_{1}, \mathrm{M}_{2}, \mathrm{M}_{3}, \mathrm{M}_{4}$ \\
Petición acceso cobot & $\mathrm{P}_{1}, \mathrm{P}_{2}, \mathrm{P}_{3}, \mathrm{P}_{4}$ \\
Uso del cobot & $\mathrm{U}_{1}, \mathrm{U}_{2}, \mathrm{U}_{3}, \mathrm{U}_{4}$ \\
Semáforo cobot & $\mathrm{C}$ \\
\hline
\end{tabular}

Una vez definidas las señales, se asignan diferentes prioridades a cada una de las máquinas, dependiendo del tipo de pieza que se esté mecanizando y de los intereses del usuario. Por tanto, la asignación de prioridades es dinámica. Como ejemplo, existe una tarea que lleva una pieza de una máquina a otra y pone una pieza nueva en la primera de ellas (ver Figura 5). Esta tarea sería la de mayor prioridad puesto que supone que dos máquinas van a estar paradas mientras se realiza. Asimismo, se ha diseñado un soporte que va incorporado en el $A M R$ para colocar la pieza mientras se mueve de una posición a otra.

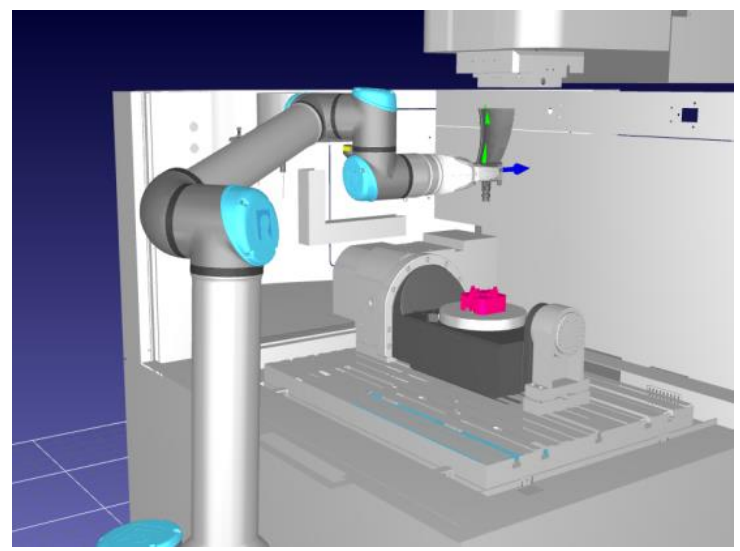

Figura 5: Cambio de pieza por el robot colaborativo

Tras definir las diferentes tareas y las prioridades de éstas, se realiza el programa que va a funcionar como semáforo de herencia de prioridades y va a dar paso a que las diferentes máquinas hagan uso del recurso compartido. Este programa será ejecutado cada vez que una de las máquinas active la señal de petición de acceso al recurso compartido. En caso de que este recurso esté liberado se dará paso a la tarea más prioritaria, y en caso de estar ocupado, la tarea queda bloqueada en la cola.

Debido a esto, se puede presentar un problema de optimización de uso de las máquinas, que sucede al producirse el bloqueo de las tareas que quieren acceder a un recurso que está ocupado. Para solucionar esto, en cada una de las máquinas, la tarea que accede al recurso compartido se ejecuta en un hilo de ejecución paralelo al del código propio de la máquina (que realiza el proceso de electroerosión). Este hilo paralelo es llamado desde el mismo programa de código propio, pero permite que la máquina siga mecanizando la pieza mientras espera a que el recurso compartido se libere. Es decir, la máquina solicita uso del recurso compartido, pero sigue ejecutando su propio código mientras espera a que el recurso se libere.

Por último, cuando una tarea va a liberar el recurso compartido, se comprueba qué máquinas están esperando para acceder al recurso y se da paso a la más prioritaria de ellas.

Este semáforo basado en herencia de prioridades se basa en el Immediate Ceiling Priority Protocol $(I C P P)$, con la única diferencia de que, si una tarea quiere acceder a un recurso compartido que está siendo utilizado, sigue ejecutando su código propio (mecanizado) en lugar de esperar a que el recurso sea liberado.

Para realizar este sistema de semáforo basado en prioridades, cuyo objetivo es que el tiempo que cada una de las máquinas está parada sea el mínimo, es necesario calcular los tiempos de mecanizado de las máquinas, el tiempo de acceso al recurso compartido y el tiempo máximo de espera para acceder al recurso. Este cálculo determinaría el mejor momento para hacer la petición de acceso al recurso compartido con suficiente antelación como para que las máquinas no se queden sin electrodos nuevos dentro de los armarios y se tenga que detener el proceso de fabricación.

\section{CONCLUSIONES}

En este trabajo se presenta el desarrollo de una aplicación de machine tending en máquinas de electroerosión mediante un robot colaborativo.

Para ello, se realiza un estudio de las diferentes tareas en las que el cobot puede asistir a la máquina. Tras diseñar los diversos elementos que ayudan al robot a realizar estas tareas, se programa en RoboDK la simulación de las mismas en un escenario con una máquina SEDM.

Una vez terminado esto, se hace una comparativa entre la vida útil de los electrodos y el tiempo necesario para que el $A M R$ reponga los electrodos de los armarios de las $S E D M$. Al ver que el cobot tiene tiempos muertos muy grandes, se llega a la conclusión de que el cobot tiene capacidad suficiente para asistir a más máquinas. Por ello, se construye un escenario con 4 máquinas en el que se implementa un semáforo basado en herencia prioridades para el acceso al recurso compartido (robot colaborativo). 


\section{Agradecimientos}

Este trabajo ha sido financiado por el proyecto Joint Action towards digital transformation (JANO) Ref. IDI-2019-1028 y parcialmente por el proyecto GUI19/045 de la UPV/EHU.

\section{English summary}

\section{COLLABORATIVE ROBOT (COBOT) APPLICATION AS AN ASSISTANT IN EDM MACHINES}

\begin{abstract}
Collaborative robots, also known as cobots, are industrial robots designed to respond to high variability and more flexible production processes. This kind of robots are very suitable for Machine Tending tasks, assisting different types of machine tool applications and allowing automation in production processes. This paper presents the design of a robotic cell where collaborative robots are integrated in order to respond to the assistance of electrodes in different EDM machines without stopping the machining process and collaborating on the user's tasks. RoboDK has been the programming tool chosen to implement the simulation.
\end{abstract}

Keywords: Collaborative robotics, cobot, automation, electrical discharge machining, EDM, RoboDK.

\section{Referencias}

[1] K. Ho and S. Newman, "State of the art electrical discharge machining (EDM)", International Journal of Machine Tools \& Manufacture, vol. 43, pp. 1287-1300, 2003.

[2] J. Krüger, T. Lien and A. Verl, "Cooperation of human and machines in assembly lines", CIRP Annals - Manufacturing Technology, vol. 58, pp. 628-646, 2009.

[3] E. Matheson, R. Minto, E. G. G. Zampieri, M. Faccio and G. Rosati, "Human-Robot
Collaboration in Manufacturing Applications: A Review", Robotics, vol. 8, no. 100, 2019.

[4] I. Maurtua, A. Ibarguren, J. Kildal, L. Susperregi and B. Sierra, "Human-Robot collaboration in industrial applications: Safety, interaction and trust", International Journal of Advanced Robotic Systems, pp. 1-10, 2017.

[5] B. Rooks, "Machine tending in the modern age", Industrial Robot: An International Journal, vol. 30, no. 4, pp. 313-318, 2003.

[6] B. Rooks, «Robot Automation in the Mould Shop», Industrial Robot, vol. 25, no 4, pp. 259261, 1998.

[7] M. Safeea, P. Neto and R. Bearee, "On-line collision avoidance for collaborative robot manipulators by adjusting off-line generated paths: An industrial use case", Robotics and Autonomous Systems, vol. 119, pp. 278-288, 2019.

[8] H. Zeng, B. Lim, G. Phua, Z. Ding, L. Aw, T. Tan, X. Li, K. Goh, P. Hee, Y. Gog and K. Yuen, "Electrode Verification and Management System for Automation of EDM Process in Mould Industry", in IECON Proceedings (Industrial Electronics Conference), 2005.

[9] UR10e Collaborative Industrial Robot - Payload up to $12,5 \mathrm{~kg}$. Link

[10] AMRs vs. AGVs: The Difference Between a Robot and a Guided Vehicle. Link

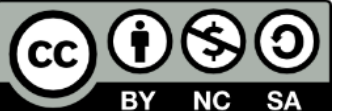
(C) 2021 by the authors. Submitted for possible open access publication under the terms and conditions of the Creative Commons Attribution CC BY-NC-SA 4.0 license (https://creativecommons.org/licenses/byncsa/4.0/deed.es). 